\title{
Miranda
}

Revue pluridisciplinaire du monde anglophone /

Multidisciplinary peer-reviewed journal on the English-

speaking world

$12 \mid 2016$

Mapping gender. Old images ; new figures

\section{Les horizons de la musique anglaise a cappella : interview de Geoffroy Heurard, baryton-basse et directeur musical de l'ensemble vocal Perspectives}

\author{
Nathalie Vincent-Arnaud
}

\section{(2) OpenEdition}

Journals

Édition électronique

URL : http://journals.openedition.org/miranda/8504

DOI : 10.4000/miranda.8504

ISSN : 2108-6559

\section{Éditeur}

Université Toulouse - Jean Jaurès

\section{Référence électronique}

Nathalie Vincent-Arnaud, « Les horizons de la musique anglaise a cappella : interview de Geoffroy Heurard, baryton-basse et directeur musical de l'ensemble vocal Perspectives », Miranda [En ligne], 12 I 2016, mis en ligne le 01 mars 2016, consulté le 16 février 2021. URL : http://journals.openedition.org/ miranda/8504; DOI : https://doi.org/10.4000/miranda.8504

Ce document a été généré automatiquement le 16 février 2021.

\section{cc)}

Miranda is licensed under a Creative Commons Attribution-NonCommercial-NoDerivatives 4.0 International License. 


\title{
Les horizons de la musique anglaise a cappella : interview de Geoffroy Heurard, baryton-basse et directeur musical de l'ensemble vocal Perspectives
}

\author{
Nathalie Vincent-Arnaud
}

1 William Blake, William Byrd, William Cornysh, Orlando Gibbons, Claudio Monteverdi, Henry Purcell, John Tavener, The Beatles, The King's Singers, Thomas Tomkins

2 Né en 2011 et réunissant cinq chanteurs issus de formations classiques, l'ensemble Perspectives pratique une musique a cappella tout à la fois rigoureuse et chatoyante, et délibérément décloisonnée. Cette approche qui embrasse plusieurs siècles de musique et se joue des étiquettes "musique savante" et "musique populaire" étant résumée dans une des rubriques de leur site internet : "Franchir les frontières, instaurer un dialogue entre les styles" (http://www.ensembleperspectives.com/fr/ensemble).

Habitués à sillonner la France, notamment à l'occasion de festivals (Festival de Radio France à Montpellier, Musicales en Normandie, Ombres et Lumières à Clairvaux), l'ensemble met fréquemment à l'honneur dans sa programmation la musique anglaise ou américaine d'époques et de styles divers. Le magnifique concert "Musiques sacrées à travers les âges" donné par cet ensemble dans le cadre du Festival Musique en Albret en juillet 2015 a permis de révéler un très vaste répertoire anglo-saxon allant de Byrd et Purcell à Tavener en passant par quelques incursions en terres pop et jazz, également nourricières de ces entre-deux dont participe aussi un attrait certain pour l'imaginaire poétique comme le montre l'intitulé d'un des programmes directement emprunté à Blake, "Songs of Experience" (http://www.ensembleperspectives.com/fr/programmes) 
Nathalie Vincent-Arnaud: "Perspectives » est un mot chargé de significations multiples. Pourquoi le choix de ce nom pour votre ensemble et quelles sont, à votre avis, les significations de ce terme les plus représentées par votre démarche artistique?

Geoffroy Heurard: Oui, c'est un mot chargé de multiples sens. Nous avons choisi le terme de Perspectives tout d'abord pour ce qu'il signifiait en termes de ligne, de point de fuite, en termes d'architecture. En effet nous nous attelons à bâtir, à construire un son avec exigence.

Ce terme évoque également une ouverture, un large champ propre à l'exploration de notre répertoire musical qui est un immense éventail.

Perspectives nous semblait aussi un mot illustrant parfaitement l'alliance entre le passé - ce terme apparait au 16ème siècle à la renaissance avec la peinture, les nuances qu'elle permet, ses dégradés - et l'époque moderne ou contemporaine où l'on parle de perspectives dans tous les domaines de la société. Les correspondances entre l'architecture, la peinture et la musique sont en effet nombreuses. Ainsi parlons-nous de la construction d'un son, de la couleur d'un accord mais aussi de nuances, d'arche sonore... Enfin, le mot Perspectives porte en lui-même la notion de projection donc de futur.

NVA : Votre répertoire fait la part belle à la musique anglaise, du Moyen Âge (William Cornysh) à l'époque contemporaine (John Tavener) en passant évidemment par le baroque (Purcell). Existe-t-il selon vous des particularités (stylistiques, thématiques) de la musique anglaise qui la différencie des autres musiques européennes à des époques données?

$\mathrm{GH}$ : Effectivement nous travaillons beaucoup de musique anglaise. Ce répertoire notamment celui de la renaissance avec son plus grand maître William Byrd et ses nombreux disciples est infini. Il est très exigeant techniquement mais procure une joie immense lorsque nous parvenons à le servir avec transparence. Nous aimons d'ailleurs débuter nos concerts avec une pièce de la Renaissance anglaise avant de créer des ponts avec d'autres époques et styles musicaux.

NVA : Quelles sont les raisons qui vous ont amené à opter pour le format a cappella?

GH : L'art a cappella est sans doute l'un des plus exigeants. C'est d'ailleurs sans doute parce qu'il ne pardonne pas la moindre approximation qu'il est aussi passionnant. En effet les couleurs que des voix peuvent créer en vibrant ensemble sur un même accord sont infinies en fonction de multiples paramètres que nous nous efforçons sans cesse de révéler. Ces paramètres sont tous humains, dépendent de nos personnalités, de notre volonté de les accorder le temps d'une oeuvre, d'un concert. La voix est l'instrument par excellence. Avec la richesse infinie de sa palette, de son expression, avec sa fragilité également elle traduit énormément. Pouvoir travailler, malaxer cette matière impalpable est pour nous une véritable passion.

NVA: Ce format évoque notamment le madrigal, dont on sait que c'est une forme particulièrement prisée en Angleterre. Quel rôle joue le madrigal dans votre répertoire en général ? Votre approche du madrigal anglais est-elle très différente de celle de son homologue italien, par exemple, et pourquoi?

GH : Là aussi tout est histoire de couleur, de vision d'un son. L'écriture d'un madrigal de Monteverdi avec sa langue italienne nous invite immédiatement à chercher davantage de brillance, de déclamation, de théâtre, de grandes arches sonores. Une pièce de Gibbons ou de Tomkins nous pousse à chercher sans doute davantage de minutie et de contrastes parfois très rapprochés. 
NVA: Vous faites des incursions dans le répertoire pop britannique (les Beatles par exemple). Existe-t-il à vos yeux une continuité, dans la musique anglaise, qui justifie cette intégration, cette abolition des frontières ? Et quel est, d'une manière générale, le sens de votre démarche?

GH : On aime en général beaucoup distinguer la musique classique - dite sérieuse - de la chanson ou la variété. Mais même si tous les chanteurs de Perspectives ont étudié la musique et le chant dans des institutions classiques (CNSM, CRR, Maitrise de RadioFrance...), nous avons également grandi avec l'univers de la chanson, de la pop, du jazz... Cette musique nous constitue tout autant et pouvoir rapprocher des styles aussi éloignés les uns des autres au sein d'un même concert nous réunit pour ainsi dire.

NVA : Cette question vient dans le prolongement de la précédente: que pensez-vous de l'opposition musique «savante» / musique «populaire»? S'agit-il d'un concept viable à toutes les époques?

GH : A Perspectives nous pensons davantage qu'il y a la bonne musique et la moins bonne, parfois la mauvaise... C'est ce dont nous voudrions témoigner dans nos concerts.

NVA: Vous avez intégré à votre répertoire le superbe morceau «The Lamb» de John Tavener (disparu en 2013), inspiré par le poème éponyme de Blake. Ce morceau a un pouvoir émotionnel extraordinaire, dans sa structure, dans la consonance du texte et de la musique, et il représente pour cela un moment très fort dans un concert; comment le ressentez-vous personnellement?

GH : C'est l'exemple typique d'œuvre que nous aimons enchaîner après une pièce de Byrd en ouvrant un concert. Ainsi nous traversons plus de quatre siècles de musique en l'espace de quelques minutes. Et ce saut dans le temps à travers les styles et les influences nous plonge dans une belle continuité, sans la moindre rupture. Cette pièce teintée d'innocence, de simplicité, que l'on pourrait définir comme une musique du silence est un moment précieux, notamment parce qu'il nous oblige à faire preuve d'humilité et ainsi à nous retrouver sur scène comme de simples médiateurs.

NVA: Pour un ensemble vocal confronté à plusieurs langues, à leurs phonèmes, à leur articulation, que représente la langue anglaise?

GH : La langue anglaise nous oblige à faire preuve d'un travail très précis, et ce pour plusieurs raisons. L'existence des diphtongues, notamment sur des valeurs longues, nous amène fréquemment à nous poser de nombreuses questions. Par ailleurs, quand nous travaillons une pièce en langue écossaise, irlandaise, anglaise, américaine, nous essayons dans la mesure du possible de faire appel à un coach pouvant nous guider dans ses nuances et ses accents. Tous ces accents contribuent grandement à rendre toute leur saveur à ces chants traditionnels.

L'importance de partager exactement la même voyelle lorsqu'on chante dans un ensemble de musique de chambre est essentielle (encore davantage lorsqu'il s'agit d'une voyelle étrangère). Cette recherche de fusion est telle que l'un des plus grands ensembles a cappella au monde, The King's Singers, explique que c'est la raison pour laquelle ne figurent que des chanteurs anglais au sein de leur groupe. 
NVA: Comptez-vous poursuivre votre démarche de décloisonnement des genres et des époques? Quel est le sens d'une telle démarche à notre époque?

GH : Cette démarche consistant à décloisonner les genres et les époques au sein d'un concert est une grande source d'énergie pour les membres de Perspectives. En effet cet exercice est délicat car il nous oblige à changer de peau et de couleur à maintes reprises au sein d'un programme. Mais au-delà de ce défi toujours renouvelé cela nous procure une fraîcheur et un bonheur unique. Le public accueille ce type de concert avec enthousiasme et cela nous incite à poursuivre dans cette optique.

INDEX

Keywords : a cappella, English music, perspectives, voice

Thèmes : Music

Mots-clés : a cappella, musique anglaise, perspectives, voix

\section{AUTEUR}

\section{NATHALIE VINCENT-ARNAUD}

Professeur

Université Toulouse-Jean Jaurès

nathalie.vincent-arnaud@univ-tlse2.fr 